\title{
Pessoas idosas institucionalizadas, transtornos depressivos e questões odontológicas: qual o estado da arte?
}

\author{
Institutionalized old people, depressive disorders, and dental issues: what is the state of the art?
}

Luiza Guilhermina de Oliveira Lopes' (ID Camila Mello dos Santos ${ }^{2}$ (D) Alexandre Fávero Bulgarelli ${ }^{3}$ (D)

\section{Resumo}

Objetivo: Fazer um mapeamento e uma discussão sobre o conhecimento científico envolvendo o objeto de pesquisa Condições de saúde bucal e depressão em idosos institucionalizados. Método: Revisão de Escopo do tipo mapeamento da literatura. O mapeamento dos dados selecionados foi feito pela técnica de sistematização de dados por meio da Análise de Conteúdo Somativa na perspectiva de Temas Manifestos nos textos. Após as exclusões foram selecionados 27 artigos. Resultados: Com a análise dos artigos foi possível dividi-los em dois temas. Todos os continentes possuem publicações acerca do tema. Sobre sua metodologia, muitas pesquisas com desenho de estudos dedutivos foram realizadas e poucas pesquisas foram desenvolvidas com métodos indutivos. Conclusão: O presente estudo identificou que existe uma interlocução entre algumas condições de saúde bucal (xerostomia e perda dentária) e prevalência de transtornos depressivos em idosos institucionalizados.

\section{Abstract}

Objective: To map and discuss scientific knowledge involving the research object Oral health conditions and depression in institutionalized old people. Method: Scope review of the literature mapping type. The mapping of the selected data was done using the data systematization technique through the Summative Content Analysis from the perspective of Manifest Themes in the texts. After the exclusions, 27 articles were selected. Results: With the analysis of the articles it was possible to divide them into two themes. All continents have publications on the topic. Regarding its methodology, a lot

Palavras-chave: Instituições de Longa Permanência para idosos. Saúde Bucal. Depressão. Literatura de Revisão como Assunto.
Keywords: Homes for the Aged. Oral Health. Depression. Review Literature as Topic.

\footnotetext{
Universidade Federal do Rio Grande do Sul, Faculdade de Odontologia. Porto Alegre, RS, Brasil.

2 Universidade Federal do Rio Grande do Sul, Departamento de Odontologia Preventiva e Social, Programa de Pós-Graduação em Odontologia. Porto Alegre, RS, Brasil.

3 Universidade Federal do Rio Grande do Sul, Departamento de Odontologia Preventiva e Social, Programa de Pós-Graduação em Saúde Coletiva. Porto Alegre, RS, Brasil.
}

Os autores declaram não haver conflito na concepção deste trabalho.

Não houve financiamento para a execução deste trabalho. 
of research with the design of deductive studies was carried out and few researches were developed with inductive methods. Conclusion: The present study identified that there is a communication between some oral health conditions (xerostomia and tooth loss) and the prevalence of depressive disorders in institutionalized old people.

\section{INTRODUÇÃO}

Os últimos 30 anos apresentam um cenário de mudanças significativas na composição familiar brasileira. Observou-se diminuição na taxa de fecundidade e a consequente redução do tamanho médio das famílias ${ }^{1}$. Além disso, a pirâmide etária brasileira revelou fenômenos como o aumento da expectativa de vida e, portanto, a tendência de envelhecimento da população. Segundo as estimativas do Instituto Brasileiro de Geografia e Estatística (IBGE) ${ }^{2}$ de 2018, houve um aumento de $18 \%$ na população de pessoas idosas na última década. Em consequência a essas transformações nas características da população, o perfil das doenças mais prevalentes do país também se altera, sobrevindo um padrão maior de doenças crônicas ${ }^{3}$.

Dentre as doenças crônicas, pode-se destacar a depressão, que afeta aproximadamente 264 milhões de pessoas em todo o mundo. Os transtornos depressivos em pessoas idosas aparecem no contexto de outras doenças crônicas, enfermidades incapacitantes e problemas familiares ${ }^{4}$. Além disso, fatores como história pessoal ou familiar de distúrbios psiquiátricos, igualmente, podem contribuir para o desenvolvimento, como apontam os dados revelados pela Organização Mundial da Saúde, que mostram que a depressão atinge cerca de $7 \%$ da população idosa, que abrange pessoas acima de 60 anos de idade 4 . Sabe-se que existe diferença entre a prevalência de doenças depressivas em idosos não-institucionalizados e idosos institucionalizados, sendo que idosos institucionalizados têm maior prevalência de doenças depressivas ${ }^{5}$. Transtornos depressivos em idosos podem levar à perda de peso, ao agravo da qualidade de vida, à alteração da autoestima, além de causarem aumento da utilização de serviços de saúde e a piora da saúde bucal ${ }^{6,7}$.

A saúde bucal de idosos, institucionalizados ou não, é fundamental para uma satisfatória qualidade de vida dessa população ${ }^{8,9}$. A manutenção de dentes remanescentes e a capacidade de oclusão são importantes para prevenir incapacidades funcionais e manter uma satisfatória qualidade de vida ${ }^{10}$. Sintomas depressivos em idosos podem ser determinantes de cáries o que mostra que a depressão pode afetar a saúde bucal de idosos ${ }^{11,12}$. Idosos institucionalizados são mais acometidos de transtornos depressivos e, nesse processo acredita-se que a prevalência de problemas bucais em idosos institucionalizados seja um importante indicador relacionado a depressão nesse grupo de idosos ${ }^{13,14,15}$.

Mediante o pressuposto de que condições relacionadas à saúde bucal possam estar associadas a condições sugestivas de depressão em idosos que se encontram institucionalizados, é fundamental mapear as evidências disponíveis para contribuir com o conhecimento científico na área de geriatria e gerontologia. Assim, o objetivo desta revisão de escopo foi mapear e identificar a produção científica envolvendo o seguinte objeto de pesquisa "Condições de saúde bucal e depressão em idosos institucionalizados".

\section{MÉTODO}

Para a realização do presente estudo sobre o estado da arte ${ }^{16}$, envolvendo a intersecção de assuntos como condições de saúde bucal e depressão em idosos institucionalizados torna-se fundamental realizar uma densa busca bibliográfica devido à amplitude do tema. Deste modo, o presente estudo propõe desenvolver uma Revisão Sistemática de Escopo do tipo mapeamento da literatura ${ }^{17,18}$. Em uma revisão de escopo, o foco é a busca do estado do conhecimento científico sobre um tema, realizada por meio de análise e discussão geral das pesquisas científicas realizadas e publicadas ${ }^{17}$. Assim, a presente revisão de literatura propõe-se a responder à seguinte questão de pesquisa: Qual o estado da arte envolvendo a interface entre questões sobre saúde bucal e depressão em pessoas idosas que se encontram institucionalizadas? 
O mapeamento dos dados selecionados para a pesquisa, desenvolvido pela técnica de sistematização de dados de Análise de Conteúdo Somativa, foi organizado na perspectiva de Temas Manifestos nos textos ${ }^{19}$. Em sequência, foi realizada a síntese, a apresentação e a descrição dos resultados de forma discursiva e temática ${ }^{17}$ organizada conforme demonstrado no Quadro 1.

Realizou-se a busca bibliográfica em bases de dados nacionais e internacionais de acesso livre por meio da plataforma Biblioteca Virtual em Saúde (BVS) com acesso livre via https://pesquisa.bvsalud.org/portal/ decs-locator/?lang=pt. A referida plataforma inclui as seguintes bases de dados da literatura: Literatura Latino-Americana e do Caribe em Ciências da Saúde (LILACS), Índice Bibliográfico Español en Ciencias de la Salud(IBECS), Medical Literature Analysis and Retrieval System Online (MEDLINE) e Scientific Electronic Library
Online (SciELO). Em todas as bases de dados acessadas utilizou-se o operador booleano $A N D$ em descritores controlados via Medical Terms (MeSH) para o resgate de evidências científicas em nível mundial.

A busca ocorreu de 12 de março a 22 de abril de 2020. Em semelhança ao estudo de Mota et al. ${ }^{20}$, foram utilizados descritores controlados em português, pois a plataforma de busca BVS recupera publicações em qualquer idioma por meio de descritores em português, inglês ou espanhol. Nesse processo, cabe destacar que a seleção do material foi efetuada por dois pesquisadores, de forma individual e independente, e houve um consenso em relação aos artigos selecionados. O processo de busca e seleção dos estudos desta revisão está apresentado no fluxograma (Figura 1), segundo checklist adaptado do Preferred Reporting Items for Systematic Reviews and Meta-Analyses (PRISMA) ${ }^{21}$.

Quadro 1. Construção dos temas envolvendo o estado da arte. Saúde bucal e sugestão de depressão em pessoas idosas institucionalizadas. 2020.

\begin{tabular}{|l|l|}
\hline Temas & Subtemas \\
\hline $\begin{array}{l}\text { 1-Condição de saúde bucal: } \\
\text { ILPI, } \\
\text { transtornos depressivos e } \\
\text { pluralidades }\end{array}$ & $\begin{array}{l}\text { Xerostomia, disfagia, lesões de mucosas e depressão mastigação, condição bucal e } \\
\text { comprometimento cognitivo, disfagia, necessidade de treinamento multiprofissional e } \\
\text { acesso a assistência odontológica } \\
\text { Cortisol na saliva, ferramentas de diagnóstico depressão } \\
\text { Histórias de vida, necessidade de saúde bucal de idosos institucionalizados } \\
\text { Habilidade mastigatórias, implantes, condições de saúde bucal e qualidade de vida }\end{array}$ \\
\hline $\begin{array}{l}\text { 2-Depressão em idosos } \\
\text { institucionalizados: } \\
\text { medicalização, } \\
\text { condições de saúde bucal e } \\
\text { subjetividades }\end{array}$ & $\begin{array}{l}\text { Medicamentos, disfagia, depressão e comportamentos } \\
\text { Depressão na transição de vida para asilo } \\
\text { Cuidado interprofissional, atividades lúdicas, exercícios físicos, intervenção nutricional } \\
\text { e cuidados da depressão } \\
\text { Atividades e cuidados sociais e redução de institucionalização } \\
\text { Ações de prevenção da depressão } \\
\text { Acesso a serviços de saúde bucal } \\
\text { Câncer, perda dentária e depressão }\end{array}$ \\
\hline
\end{tabular}




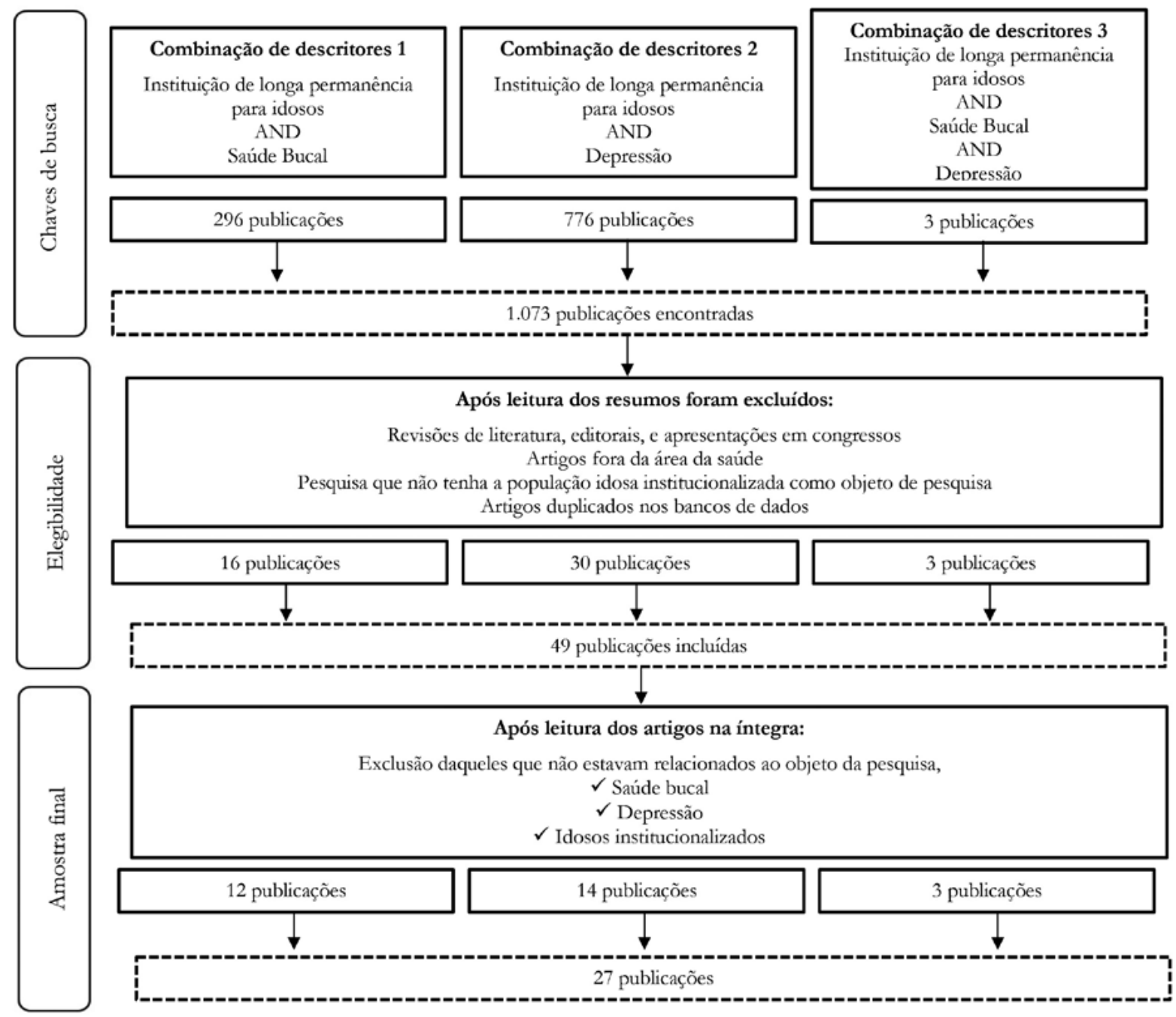

Figura 1. Idosos institucionalizados, questões odontológicas e transtornos depressivos. Fluxograma do processo de seleção dos estudos, adaptado do PRISMA $21,2020$.

Deste modo foram incluídos artigos: com ano de publicação livre, para registro da primeira evidência publicada sobre o tema; artigos que tivessem seus títulos, resumos e artigos na íntegra disponibilizados nos idiomas português, inglês ou espanhol; artigos que estivessem relacionados com a temática proposta, e que fossem acessados pelos descritores utilizados. Foram excluídos artigos de revisão de literatura, relatos de caso, editoriais e manuais, relatórios e protocolos clínicos de cuidados. Da amostra final, foram analisados dados de caracterização da produção como: ano de publicação; país de realização da pesquisa; objetivo do estudo; abordagem metodológica; assunto relativo à depressão e à saúde bucal.
As estratégias de busca permitiram identificar 1.073 artigos. A leitura com análise dos 49 títulos e resumos, com a finalidade de selecionar os artigos que fossem pertinentes à pergunta de pesquisa, resultou na manutenção de 27 artigos, que tinham como fenômeno de interesse o estudo dos temas idosos institucionalizados, questões odontológicas e transtornos depressivos. Cada artigo foi lido na íntegra pelos pares e foram estruturados nos seguintes temas: Condição de saúde bucal: Instituições de Longa Permanência (ILPI), transtornos depressivos e pluralidades e Depressão em idosos institucionalizados: medicalização, condições de saúde bucal e subjetividades. 


\section{RESULTADOS}

Em resposta à questão da presente pesquisa, observou-se que houve uma heterogeneidade nos subtemas dos artigos acessados bem como nas características dos estudos publicados.

O primeiro estudo acessado foi publicado no ano de 2009 e é brasileiro. A última publicação foi no ano de 2019 e tem o Brasil e os Estados Unidos como origens da pesquisa. Neste contexto, é possível destacar que dentre todos os estudos analisados, seis estudos têm origem no Brasil e, destes, três trazem a interlocução dos assuntos depressão e saúde bucal em idosos institucionalizados (Tabelas 1 e 2).
Dentre os estudos observou-se diversos objetivos como descrição da condição de saúde bucal e o status dos implantes dentários em idosos institucionalizados até objetivos mais associados a análise de risco de demência com uso de anticolinérgico (Tabelas 1 e 2). Quanto às metodologias utilizadas nos estudos analisados, foi possível observar que muitas pesquisas com desenho de estudo dedutivo foram realizadas e poucas pesquisas foram desenvolvidas com métodos indutivos. Além desses aspectos, apenas um estudo, com origem Australiana utiliza de metodologia mista, o que mostra que pouco se vem pesquisando com tal método (Tabela 2).

Tabela 1. Mapeamento dos estudos incluídos na revisão de escopo categorizados no Tema 1, por ordem cronológica crescente com base no ano de publicação, 2009-2020.

\begin{tabular}{|c|c|c|c|c|}
\hline $\begin{array}{l}\text { Ano de Publicação/ } \\
\text { Autor(es)/País de } \\
\text { origem da pesquisa }\end{array}$ & Objetivo do estudo & $\begin{array}{l}\text { Abordagem } \\
\text { metodológica }\end{array}$ & $\begin{array}{l}\text { Assunto } \\
\text { relativo à } \\
\text { depressão } \\
\text { abordado }\end{array}$ & $\begin{array}{l}\text { Assunto relativo à } \\
\text { saúde bucal abordado }\end{array}$ \\
\hline $\begin{array}{l}\text { 2009/De Mello } \\
\text { ALSF, Padilha } \\
\text { DMP/ } \\
\text { Brasil }^{22}\end{array}$ & $\begin{array}{l}\text { Identificar as características da } \\
\text { assistência à saúde bucal para } \\
\text { idosos institucionalizado. }\end{array}$ & $\begin{array}{l}\text { Qualitativa/ } \\
\text { Análise temática }\end{array}$ & - & $\begin{array}{l}\text { Higiene bucal, } \\
\text { assistência } \\
\text { odontológica }\end{array}$ \\
\hline $\begin{array}{l}\text { 2009/Ferreira RC, } \\
\text { et al. / } \\
\text { Brasil }^{23}\end{array}$ & $\begin{array}{l}\text { Descrever a saúde bucal dos idosos } \\
\text { institucionalizado quanto à cárie } \\
\text { dentária, higiene bucal e doença } \\
\text { periodontal. }\end{array}$ & $\begin{array}{l}\text { Quantitativa/ } \\
\text { Estudo descritivo }\end{array}$ & - & $\begin{array}{l}\text { Higiene bucal, cárie } \\
\text { dentária coronária e } \\
\text { radicular e doença } \\
\text { periodontal }\end{array}$ \\
\hline $\begin{array}{l}\text { 2009/Haumschild } \\
\text { MS, Haumschild } \\
\text { RJ/ } \\
\text { Estados Unidos }^{24}\end{array}$ & $\begin{array}{l}\text { Avaliar a importância dos } \\
\text { cuidados com a saúde bucal em } \\
\text { longo prazo e sua relação com a } \\
\text { manutenção da saúde geral. }\end{array}$ & $\begin{array}{l}\text { Qualitativa/ } \\
\text { Análise narrativa }\end{array}$ & - & $\begin{array}{l}\text { Higiene bucal e } \\
\text { periodontite }\end{array}$ \\
\hline $\begin{array}{l}\text { 2009/Isaksson R, } \\
\text { et al. / } \\
\text { Suécia }^{25}\end{array}$ & $\begin{array}{l}\text { Investigar a saúde bucal e o } \\
\text { status dos implantes orais em } \\
\text { pacientes que recebem cuidados } \\
\text { a longo prazo ou que são } \\
\text { institucionalizados, todos com } \\
\text { próteses. }\end{array}$ & $\begin{array}{l}\text { Quantitativa/ } \\
\text { Estudo coorte }\end{array}$ & - & $\begin{array}{l}\text { Estomatite, assistência } \\
\text { odontológica para } \\
\text { idosos, edentulismo, } \\
\text { prótese dentária } \\
\text { suportada por implante } \\
\text { e placa dentária }\end{array}$ \\
\hline $\begin{array}{l}\text { 2009/Kim HY, et } \\
\text { al. / } \\
\text { Coréia do Sul }{ }^{26}\end{array}$ & $\begin{array}{l}\text { Avaliar a associação entre } \\
\text { capacidade mastigatória e } \\
\text { qualidade de vida relacionada à } \\
\text { saúde bucal, associando a outros } \\
\text { fatores. }\end{array}$ & $\begin{array}{l}\text { Quantitativo/ } \\
\text { Estudo descritivo } \\
\text { com dados } \\
\text { secundários }\end{array}$ & - & $\begin{array}{l}\text { Capacidade } \\
\text { mastigatória, } \\
\text { edentulismo e } \\
\text { autopercepção em } \\
\text { saúde bucal }\end{array}$ \\
\hline
\end{tabular}


Continuação da Tabela 1

\begin{tabular}{|c|c|c|c|c|}
\hline $\begin{array}{l}\text { Ano de Publicação/ } \\
\text { Autor(es)/País de } \\
\text { origem da pesquisa }\end{array}$ & Objetivo do estudo & $\begin{array}{l}\text { Abordagem } \\
\text { metodológica }\end{array}$ & $\begin{array}{l}\text { Assunto } \\
\text { relativo à } \\
\text { depressão } \\
\text { abordado }\end{array}$ & $\begin{array}{l}\text { Assunto relativo à } \\
\text { saúde bucal abordado }\end{array}$ \\
\hline $\begin{array}{l}\text { 2010/Bush HM, } \\
\text { et al./ } \\
\text { Estados Unidos }{ }^{27}\end{array}$ & $\begin{array}{l}\text { Avaliar o estado de saúde bucal de } \\
\text { idosos. }\end{array}$ & $\begin{array}{l}\text { Quantitativo/ } \\
\text { Estudo descritivo } \\
\text { com dados } \\
\text { secundários }\end{array}$ & - & $\begin{array}{l}\text { Estado geral de } \\
\text { saúde bucal (dor, } \\
\text { sangramento gengival } \\
\text { e perda dentária, uso } \\
\text { de próteses) e acesso a } \\
\text { serviços odontológicos }\end{array}$ \\
\hline $\begin{array}{l}\text { 2010/De Visschere } \\
\text { L, et al. J./ } \\
\text { Bélgica }^{28}\end{array}$ & $\begin{array}{l}\text { Comparar uma implementação } \\
\text { supervisionada versus uma não } \\
\text { supervisionada de uma diretriz de } \\
\text { saúde bucal }\end{array}$ & $\begin{array}{l}\text { Quantitativo/ } \\
\text { Ensaio Clínico } \\
\text { Randomizado }\end{array}$ & - & Higiene Bucal \\
\hline $\begin{array}{l}\text { 2011/Ozkan Y, } \\
\text { et al / } \\
\text { Turquia }^{29}\end{array}$ & $\begin{array}{l}\text { Determinar o estado de } \\
\text { saúde, além das necessidades } \\
\text { de tratamento, em idosos } \\
\text { institucionalizados. }\end{array}$ & $\begin{array}{l}\text { Quantitativo/ } \\
\text { Estudo descritivo }\end{array}$ & - & $\begin{array}{l}\text { Acesso a serviços } \\
\text { odontológicos, estado } \\
\text { dentário atual, uso e } \\
\text { estado de próteses, } \\
\text { higiene bucal e } \\
\text { edentulismo. }\end{array}$ \\
\hline $\begin{array}{l}\text { 2012/Mozafari } \\
\text { PM, et al/ } \\
\text { Irã } \\
\end{array}$ & $\begin{array}{l}\text { Determinar a prevalência de } \\
\text { lesões da mucosa oral em idosos } \\
\text { institucionalizados. }\end{array}$ & $\begin{array}{l}\text { Quantitativo/ } \\
\text { Estudo analítico }\end{array}$ & - & Lesões da mucosa oral \\
\hline $\begin{array}{l}\text { 2014/Pretty IA/ } \\
\text { Inglaterra }^{31}\end{array}$ & $\begin{array}{l}\text { Abordar a avaliação das } \\
\text { necessidades de saúde bucal } \\
\text { com base no curso da vida } \\
\text { e vulnerabilidade em idosos } \\
\text { institucionalizados. }\end{array}$ & $\begin{array}{l}\text { Quantitativo/ } \\
\text { Estudo descritivo }\end{array}$ & $\begin{array}{l}\text { Depressão } \\
\text { como } \\
\text { comorbidade }\end{array}$ & $\begin{array}{l}\text { Uso de próteses, } \\
\text { presença de dor, acesso } \\
\text { a serviços de saúde } \\
\text { bucal, autocuidado em } \\
\text { saúde bucal }\end{array}$ \\
\hline $\begin{array}{l}\text { 2018/Cocco F, } \\
\text { et al/ } \\
\text { Itália }^{32}\end{array}$ & $\begin{array}{l}\text { Avaliar a prevalência e gravidade } \\
\text { da perda dentária em idosos } \\
\text { institucionalizados e associar } \\
\text { outros dados. }\end{array}$ & $\begin{array}{l}\text { Estudo } \\
\text { quantitativo } \\
\text { observacional } \\
\text { descritivo } \\
\text { seccional com } \\
\text { dados primários e } \\
\text { secundários }\end{array}$ & $\begin{array}{l}\text { Depressão } \\
\text { como } \\
\text { comorbidade }\end{array}$ & Perda Dentária \\
\hline $\begin{array}{l}\text { 2018/Machado } \\
\mathrm{ACB} / \\
\text { Brasil }^{33}\end{array}$ & $\begin{array}{l}\text { Analisar as concentrações } \\
\text { de cortisol salivar de idosos } \\
\text { institucionalizados e não } \\
\text { institucionalizados e verificar } \\
\text { as condições de saúde bucal e } \\
\text { dependência física. }\end{array}$ & $\begin{array}{l}\text { Estudo } \\
\text { transversal, } \\
\text { descritivo e } \\
\text { analítico }\end{array}$ & $\begin{array}{l}\text { Depressão } \\
\text { como objeto } \\
\text { central }\end{array}$ & $\begin{array}{l}\text { Uso e necessidade de } \\
\text { prótese dentária }\end{array}$ \\
\hline $\begin{array}{l}\text { 2019/Recker E, } \\
\text { et al/ } \\
\text { Brasil e Estados } \\
\text { Unidos }^{34}\end{array}$ & $\begin{array}{l}\text { Descrever e comparar xerostomia } \\
\text { e saúde geral em idosos } \\
\text { institucionalizados. }\end{array}$ & $\begin{array}{l}\text { Estudo } \\
\text { quantitativo } \\
\text { descritivo com } \\
\text { dados secundários }\end{array}$ & $\begin{array}{l}\text { Depressão } \\
\text { como } \\
\text { comorbidade }\end{array}$ & Xerostomia \\
\hline
\end{tabular}


Tabela 2. Mapeamento dos estudos incluídos na revisão de escopo categorizados no Tema 2, por ordem cronológica crescente com base no ano de publicação, 2009-2020.

\begin{tabular}{|c|c|c|c|c|}
\hline $\begin{array}{l}\text { Ano de Publicação/ } \\
\text { Autor(es)/País de } \\
\text { origem da pesquisa. }\end{array}$ & Objetivo do estudo & $\begin{array}{l}\text { Abordagem } \\
\text { metodológica }\end{array}$ & $\begin{array}{l}\text { Assunto relativo } \\
\text { à depressão } \\
\text { abordado }\end{array}$ & $\begin{array}{l}\text { Assunto } \\
\text { relativo à saúde } \\
\text { bucal abordado }\end{array}$ \\
\hline $\begin{array}{l}\text { 2012/Cassie KM, } \\
\text { Cassie WE./ } \\
\text { Estados Unidos }\end{array}$ & $\begin{array}{l}\text { Analisar o efeito da cultura } \\
\text { e do clima sobre os sintomas } \\
\text { depressivos entre idosos } \\
\text { institucionalizados. }\end{array}$ & $\begin{array}{l}\text { Quantitativo/ } \\
\text { Estudo } \\
\text { descritivo } \\
\text { com dados } \\
\text { secundários }\end{array}$ & $\begin{array}{l}\text { Depressão como } \\
\text { objeto central }\end{array}$ & - \\
\hline $\begin{array}{l}\text { 2012/Davison TE, } \\
\text { et al./ } \\
\text { Austrália }^{36}\end{array}$ & $\begin{array}{l}\text { Examinar o uso da Escala } \\
\text { de Cornell em instalações } \\
\text { de atendimento em Sydney e } \\
\text { Melbourne, na Austrália, para } \\
\text { residentes deprimidos. }\end{array}$ & $\begin{array}{l}\text { Quantitativo/ } \\
\text { Estudo } \\
\text { descritivo }\end{array}$ & $\begin{array}{l}\text { Depressão como } \\
\text { objeto central }\end{array}$ & - \\
\hline $\begin{array}{l}\text { 2013/Bomfim FMS, } \\
\text { Chiari BM, Roque } \\
\text { FP/ } \\
\text { Brasil }{ }^{37}\end{array}$ & $\begin{array}{l}\text { Identificar os fatores associados } \\
\text { a sinais sugestivos de disfagia } \\
\text { orofaríngea em idosas } \\
\text { institucionalizadas. }\end{array}$ & $\begin{array}{l}\text { Quantitativo/ } \\
\text { Estudo } \\
\text { descritivo }\end{array}$ & $\begin{array}{l}\text { Depressão como } \\
\text { comorbidade }\end{array}$ & $\begin{array}{l}\text { Número de } \\
\text { dentes em boca }\end{array}$ \\
\hline $\begin{array}{l}\text { 2013/Drageset J, Eide } \\
\text { GE, Ranhoff AH/ } \\
\text { Noruega }^{38}\end{array}$ & $\begin{array}{l}\text { Analisar a hipótese de que } \\
\text { ansiedade ou depressão possuem } \\
\text { relação com sobrevida de } \\
\text { pacientes com e sem câncer. }\end{array}$ & $\begin{array}{l}\text { Quantitativo/ } \\
\text { Estudo de coorte }\end{array}$ & $\begin{array}{l}\text { Depressão como } \\
\text { objeto central }\end{array}$ & - \\
\hline $\begin{array}{l}\text { 2013/Underwood M, } \\
\text { et al./ } \\
\text { Reino Unido }{ }^{39}\end{array}$ & $\begin{array}{l}\text { Testar a hipótese de que um } \\
\text { programa de exercícios reduziria } \\
\text { sintomas depressivos }\end{array}$ & $\begin{array}{l}\text { Quantitativo/ } \\
\text { Ensaio Clínico } \\
\text { Randomizado }\end{array}$ & $\begin{array}{l}\text { Depressão como } \\
\text { comorbidade }\end{array}$ & - \\
\hline $\begin{array}{l}\text { 2014/Miller LM, et } \\
\text { al./ } \\
\text { Estados Unidos }^{40}\end{array}$ & $\begin{array}{l}\text { Determinar o papel de atividades } \\
\text { físicas no risco de colocação em } \\
\text { ILPI }\end{array}$ & $\begin{array}{l}\text { Quantitativo/ } \\
\text { Estudo de coorte }\end{array}$ & $\begin{array}{l}\text { Depressão como } \\
\text { comorbidade }\end{array}$ & - \\
\hline $\begin{array}{l}\text { 2014/Van Schaik DJ, } \\
\text { et al./ } \\
\text { Holanda }^{41}\end{array}$ & $\begin{array}{l}\text { Avaliar os efeitos de um } \\
\text { programa de cuidados intensivos } \\
\text { para evitar o aparecimento } \\
\text { de depressão em idosos } \\
\text { institucionalizados }\end{array}$ & $\begin{array}{l}\text { Quantitativo/ } \\
\text { Ensaio Clínico } \\
\text { Randomizado }\end{array}$ & $\begin{array}{l}\text { Depressão como } \\
\text { objeto central }\end{array}$ & - \\
\hline $\begin{array}{l}\text { 2015/Chesler J, et al./ } \\
\text { Austrália }^{42}\end{array}$ & $\begin{array}{l}\text { Investigar um programa de } \\
\text { intervenção destinado a promover } \\
\text { relacionamentos entre idosos em } \\
\text { atendimento }\end{array}$ & Estudo Misto & $\begin{array}{l}\text { Depressão como } \\
\text { comorbidade }\end{array}$ & - \\
\hline $\begin{array}{l}\text { 2016/Bali V, et al./ } \\
\text { Estados Unidos }^{43}\end{array}$ & $\begin{array}{l}\text { Analisar o risco de demência com } \\
\text { uso anticolinérgico em idosos } \\
\text { residentes em casas de repouso } \\
\text { com depressão }\end{array}$ & $\begin{array}{l}\text { Quantitativo/ } \\
\text { Estudo caso- } \\
\text { controle }\end{array}$ & $\begin{array}{l}\text { Depressão como } \\
\text { objeto central }\end{array}$ & - \\
\hline $\begin{array}{l}\text { 2016/Menezes AV, } \\
\text { et al./ } \\
\text { Brasil }^{44}\end{array}$ & $\begin{array}{l}\text { Verificar a efetividade de } \\
\text { intervenção fisioterapêutica } \\
\text { na cognição, mobilidade e } \\
\text { independência funcional de } \\
\text { idosos institucionalizados }\end{array}$ & $\begin{array}{l}\text { Quanti/ } \\
\text { Ensaio Clínico } \\
\text { Randomizado }\end{array}$ & $\begin{array}{l}\text { Depressão como } \\
\text { comorbidade }\end{array}$ & - \\
\hline
\end{tabular}


Continuação da Tabela 2

\begin{tabular}{|c|c|c|c|c|}
\hline $\begin{array}{l}\text { Ano de Publicação/ } \\
\text { Autor(es)/País de } \\
\text { origem da pesquisa. }\end{array}$ & Objetivo do estudo & $\begin{array}{l}\text { Abordagem } \\
\text { metodológica }\end{array}$ & $\begin{array}{l}\text { Assunto relativo } \\
\text { à depressão } \\
\text { abordado }\end{array}$ & $\begin{array}{l}\text { Assunto } \\
\text { relativo à saúde } \\
\text { bucal abordado }\end{array}$ \\
\hline $\begin{array}{l}\text { 2016/Mountford CG, } \\
\text { et al./ } \\
\text { Reino Unido }\end{array}$ & $\begin{array}{l}\text { Estabelecer a prevalência de } \\
\text { desnutrição e investigar a } \\
\text { efetividade de um programa de } \\
\text { intervenção nutricional }\end{array}$ & $\begin{array}{l}\text { Estudo } \\
\text { quantitativo } \\
\text { observacional } \\
\text { descritivo de } \\
\text { coorte }\end{array}$ & $\begin{array}{l}\text { Depressão como } \\
\text { comorbidade }\end{array}$ & - \\
\hline $\begin{array}{l}\text { 2018/Byers AL, Lui } \\
\text { et al./ } \\
\text { Estados Unidos }{ }^{46}\end{array}$ & $\begin{array}{l}\text { Determinar a associação entre } \\
\text { carga cumulativa de sintomas } \\
\text { depressivos e risco de colocação } \\
\text { em ILPI }\end{array}$ & $\begin{array}{l}\text { Estudo } \\
\text { quantitativo } \\
\text { observacional } \\
\text { analítico de } \\
\text { coorte }\end{array}$ & $\begin{array}{l}\text { Depressão como } \\
\text { objeto central }\end{array}$ & - \\
\hline $\begin{array}{l}\text { 2019/Ozaki T, } \\
\text { Katsumata Y, Arai A/ } \\
\text { Japão }^{47}\end{array}$ & $\begin{array}{l}\text { Investigar a associação entre } \\
\text { as mudanças no uso de drogas } \\
\text { psicotrópicas e sintomas } \\
\text { psicológicos de demência }\end{array}$ & $\begin{array}{l}\text { Estudo } \\
\text { quantitativo } \\
\text { observacional } \\
\text { analítico de } \\
\text { coorte }\end{array}$ & $\begin{array}{l}\text { Depressão como } \\
\text { comorbidade }\end{array}$ & - \\
\hline
\end{tabular}

\section{DISCUSSÃO}

Esta revisão de escopo mapeou em bases de dados nacionais e internacionais de interesse temas com idosos institucionalizados, questões odontológicas e transtornos depressivos. De acordo com o relatório World Population Prospects de 2019, estima-se que em 2050, uma a cada seis pessoas no mundo estará acima de 65 anos $^{4}$. Já no continente europeu e na américa do norte, as estimativas são diferentes. Nesses lugares, uma a cada quatro pessoas poderá ter 65 anos ou mais. Ainda, segundo o relatório, países mais pobres terão sua população com expectativa de vida sete anos menor que a média mundial ${ }^{4}$. Ao relacionarmos as estimativas do envelhecimento populacional mundial com os dados obtidos no atual trabalho, é possível analisar que os 27 artigos selecionados foram publicados em 14 países no total. E, desses países, nove estão entre os 25 mais desenvolvidos do mundo e, portanto, possuem maior expectativa de vida e maior projeção de envelhecimento da população em relação aos menos desenvolvidos ${ }^{35}$.

Nos nove países supracitados, o número de Instituições de Longa Permanência para Idosos é maior em número absoluto e relativo que o número dos outros países de origem dos artigos ${ }^{37}$. Com isso, pode-se relacionar a grande produção científica de países desenvolvidos acerca desse assunto com a porcentagem elevada da sua população idosa. Neste contexto, é importante ressaltar a relevância e a vanguarda brasileira na construção de conhecimento científico sobre os temas observados. De maneira geral, todos os continentes originaram estudos sobre a temática; e na América Latina, apenas o Brasil publicou estudos sobre o tema.

Nos artigos em que a saúde bucal foi abordada, foram citados os problemas bucais mais prevalentes em idosos, dentre eles destacam-se perda dentária, periodontite, xerostomia, lesões na mucosa oral e cárie dentária. O controle dessas doenças, além de trazer benefícios para a saúde bucal, ainda proporciona melhoria na saúde gera $1^{15}$. No entanto, o padrão epidemiológico das doenças bucais em idosos está mudando conforme ocorre o envelhecimento da população.

Em um estudo realizado com 75 idosos de uma instituição de longa permanência para idosos no Egito, a educação em saúde bucal foi abordada como um fator importante para a ampliação da qualidade de vida dos residentes. Os participantes do estudo em que foram aplicadas as ferramentas de educação demonstraram uma melhora significativa no auto cuidado da higiene bucal. Os dados do artigo referenciado demonstram que os idosos 
institucionalizados possuem, de fato, potencial para ter maior autonomia em relação aos próprios cuidados de higiene oral, visto que, em alguns artigos da amostra o cuidado da saúde bucal do idoso é considerado responsabilidade exclusiva da instituição ou da sua família ${ }^{43}$.

Considerando o tema "Condição de saúde bucal: ILPI, transtornos depressivos e pluralidades" observase que a condição de saúde bucal de uma pessoa idosa revela muito sobre sua condição geral de saúde bem como sua saúde mental ${ }^{13}$. Elucidando a saúde bucal nas questões de saúde mental e pessoas idosas institucionalizadas foram encontrados doze estudos que constituíram o conteúdo do presente tema. Muitas vezes, a literatura aponta condições precárias da saúde bucal de idosos institucionalizados e, como reflexo desse aspecto, a consequente vulnerabilidade de idosos institucionalizados ${ }^{22,23,27,31}$. A literatura mostra, também, que existe uma interlocução de achados sobre as condições de saúde bucal de idosos em idosos institucionalizados, como a xerostomia, a perda dentária e prevalência de transtornos depressivos nessa mesma populaçãa ${ }^{24,29,32,34}$. Os estudos que apontam achados sobre precárias condições bucais encontramse ligados, de certa forma, às evidências científicas sobre transtornos depressivos nessa população. Deste modo, acredita-se que a relação entre transtornos depressivos e saúde bucal é assunto a ser investigado para população de idosos em ILPI, pois o estado da arte aponta lacunas a serem investigadas. Além disso, a literatura mostra a importância em incluir o cirurgião dentista nos cuidados para prevenir agravos à saúde mental de idosos ${ }^{13}$.

Ao abordar a pluralidade de assuntos e temas de pesquisa envolvendo a questão da saúde bucal e transtornos depressivos, cabe destacar que a depressão pode afetar a saúde bucal de pessoas idosas, pois tal doença leva a negligência nos procedimentos de higiene e alimentação cariogênica o que leva a um risco aumentado de doença cárie e periodontal ${ }^{6}$. Neste contexto tem-se que idosos com depressão relatam sensação de boca seca e dor de origem bucal em prevalência maior que idosos sem transtorno depressivo?.

A presente revisão de literatura aponta que os transtornos depressivos podem ser diagnosticados por meio de cavidade bucal. Por meio da identificação de concentração de cortisol na saliva é possível o diagnóstico de depressão ${ }^{33}$. De maneira mais ampla foi possível perceber, também, que a melhoria da habilidade mastigatória com a utilização de implantes afeta diretamente na qualidade de vida ${ }^{25,26,30}$.

Estudo de Cocco et al. ${ }^{32}$, que usou ferramentas para avaliar número de dentes funcionais, presença e tipos de restaurações dentárias, presença e tipos de próteses, lesões de cárie e fatores sistêmicos como índice de massa corporal, condições de saúde mental e status nutricional. Desta forma, estudos qualitativos não estão sendo utilizados com frequência, na abordagem científica desse tema. Em estudos qualitativos, o autor busca interagir diretamente com o objeto de estudo, a fim de fazer uma análise sem dados estatísticos sobre o assunto, analisando individualmente as respostas e fazendo uma reflexão considerando a sua hipótese inicial e os resultados obtidos ${ }^{17}$. Sendo assim, acredita-se que fatores subjetivos que venham a alterar dados objetivos e estatísticos podem ser analisados de forma criteriosa respondendo à questão do presente estudo. Por conseguinte, sugere-se que metodologias qualitativas possam ser mais exploradas no que tange ao tema em estudo.

Os resultados do estudo de Machado ${ }^{33}$, apontam maiores concentrações de cortisol na saliva de idosos institucionalizados com autopercepção negativa da saúde bucal. Levando em consideração que o cortisol está associado aos níveis de estresse, percebeu-se que níveis altos de cortisol podem levar essa população ao desenvolvimento de transtornos depressivos.

O tema "Depressão em idosos institucionalizados: medicalização, condições de saúde bucal e subjetividades", traz o idoso institucionalizado para o foco da discussão entre transtornos depressivos e questões envolvendo a saúde bucal, pois a depressão em idosos institucionalizados pode levar a comprometimentos funcionais e a perda da autonomia para o autocuidado ${ }^{14}$. Além desses aspectos, idosos institucionalizados e com depressão apresentam alta prevalência de perda dental, falta de cuidados preventivos e falta de acesso a tratamentos odontológicos o que leva a uma pior saúde bucal ${ }^{15}$.

Observa-se que, ao contrário do tema anterior, o presente tema possui exemplos de estudos que 
exploram métodos de pesquisas para explorar dados quantitativos e subjetividades. Destaca-se como exemplo o estudo de Cassie et al. ${ }^{47}$, que abordou valores, crenças e expectativas dos trabalhadores de instituições como fatores que poderiam afetar diretamente a qualidade de vida e depressão dos residentes. O referido estudo obteve como um de seus resultados maior sintomatologia depressiva em idosos institucionalizados em instituições com ambiente de trabalho saudável. Uma das considerações dos autores sobre esse resultado foi o fato de que, em ambientes mais agradáveis, as características individuais dos residentes são observadas pelos profissionais com maior atenção e cuidado, desta forma, a sintomatologia depressiva torna-se mais evidente nesses locais.

Neste tema, o assunto medicalização foi abordado de forma direta em dois estudos, porém ele é recorrente como fator adicional associado a outros objetos em estudos dos temas 1 e 2 No estudo de Ozaki, Katsumata e Arai ${ }^{48}$, drogas psicotrópicas, em especial os ansiolíticos e hipnóticos, são associadas ao aumento de sintomas de demência em residentes de instituições de longa permanência. Bali et al. ${ }^{49}$, também buscaram associar mortalidade entre idosos institucionalizados com o uso concomitante de medicamentos anticolinérgicos e depressivos. Neste caso, não foram encontradas associações. A grande quantidade de estudos na literatura sobre medicalização em idosos institucionalizados pode dar-se devido ao alto número de medicamentos consumidos por essa população, em comparação com faixa etária mais jovem. No entanto, cabe ressaltar a importância de fazer comparações constantes entre idosos institucionalizados e idosos nãoinstitucionalizados, de forma que se entenda se esses estudos poderiam ser aplicados a toda população de uma região. É importante que sejam investigadas as diferenças na forma com que os medicamentos podem interagir em diferentes condições depressivas e vulnerabilidades.

O único assunto abordado relativo à saúde bucal no referido Tema 2 , foi o número de dentes em boca $^{50}$. A saúde bucal e a depressão podem estar associadas e o reduzido número de dentes é um reflexo dessa associação ${ }^{6,15,23}$. Estudos que abordam a saúde mental de idosos institucionalizados e sua relação com a saúde bucal desta população parece não ser representativos na literatura mundial. Sugerese que o baixo número de estudos no referido tema seja devido a esse fato. Desta forma questiona-se: em estudos cujo tema central é a depressão, não deveria o tema de saúde bucal ser mais estudado?

Observou-se que, no que tange aos dois temas apresentados no presente estudo, aparece de forma recorrente a associação entre aspectos socioeconômicos e capacidade cognitiva ou depressão. O estudo de Ozkan et al. ${ }^{29}$, aborda esses dois assuntos de forma enfática. Seu objetivo foi determinar o estado de saúde bucal e geral, além das necessidades, de idosos institucionalizados na cidade de Istambul. Interessante ressaltar neste caso os resultados obtidos, levando em consideração que a saúde bucal nessa população foi considerada deficiente. Um dos fatores relevantes para o estado de saúde bucal dessa população foi o preço dos procedimentos e, portanto, a falta de acesso à atendimentos odontológicos dessa população. Assim como o Brasil, a Turquia é um país emergente, isto é, país em desenvolvimento econômico, com índices de desenvolvimento humano mediano, grande população e com exportação, principalmente, de matéria prima ${ }^{24}$. De maneira geral, países emergentes partilham a característica de serem nações com grandes desigualdades sociais. Essas características podem não somente afetar a economia da nação, mas também a qualidade de vida e a saúde da sua população. Sugere-se que índices socioeconômicos mais baixos sejam considerados fatores limitantes para boas condições de saúde bucal de uma população.

É importante ressaltar as limitações do presente estudo, iniciando pela inclusão de estudos acessados por descritos em inglês e espanhol e português simultaneamente e não apenas acesso em língua Inglesa. Apesar dessa limitação, os resultados do presente estudo não foram invalidados, pois foram utilizados artigos científicos publicados em periódicos indexados. Também houve uma restrição de bases e termos utilizados. No entanto, a presente pesquisa foi baseada em referências reconhecidas internacionalmente, como Preferred Reporting Items for Systematic Reviews and Meta-Analyses (PRISMA) ${ }^{21}$. Assim, o resultado do mapeamento sobre as condições de saúde bucal e depressão em pessoas idosas institucionalizadas poderá contribuir para o conhecimento científico na área de geriatria e gerontologia. 


\section{CONCLUSÃO}

O conhecimento que vem se produzindo sobre saúde bucal, saúde mental em idosos institucionalizados é relativamente recente, reflexo da contemporaneidade do tema. Além disso, a construção desse conhecimento está associada à diversidade de estudos epidemiológicos e qualitativos buscando responder questões que envolvem a pluralidade técnica e subjetiva envolvendo a saúde mental e a saúde bucal de pessoas idosas institucionalizadas.

O presente estudo identificou que existe uma interlocução entre condições de saúde bucal como xerostomia e perda dentária e a prevalência de transtornos depressivos. A relação entre depressão e saúde bucal precisa de estudos com delineamentos longitudinais para identificar possíveis mecanismos envolvidos nessa associação em pessoas idosas institucionalizadas. Sugere-se que mais estudos sejam realizados com outras fontes de dados e com diferentes descritores para ampliar o conhecimento na área. Ademais, é fundamental o desenvolvimento de novas agendas de políticas públicas com medidas de prevenção, promoção e assistência englobando saúde bucal e mental.

Editado por: Maria Helena Rodrigues Galvão

\section{REFERÊNCIAS}

1. Núñez JG. Uneven decline in fertility rates in adolescents in 32 countries of the Region of the Americas, 1960-2019. Rev Panam Salud Publica. 2020;44:e71. Disponível em: https://doi. org/10.26633/rpsp.2020.71 .

2. Instituto Brasileiro de Geografia e Estatística [Internet]. Rio de Janeiro: IBGE; 2020 [acesso em 29 mar. 2020]. Número de idosos cresce $18 \%$ em 5 anos e ultrapassa 30 milhões em 2017. Disponível em: https://agenciadenoticias.ibge.gov.br/agencianoticias/2012-agencia-de-noticias/noticias/20980numero-de-idosos-cresce-18-em-5-anos-e-ultrapassa30-milhoes-em-2017 .

3. Massa KHC, Duarte YAO, Chiavegatto Filho ADP. Análise da prevalência de doenças cardiovasculares e fatores associados em idosos, 2000-2010. Ciênc Saúde Colet. 2019;24(1):105-14. Disponível em: https://doi. org/10.1590/1413-81232018241.02072017 .

4. World Health Organization [Internet]. Geneva: WHO; 2019 [acesso em 11 mar. 2021]. World population prospects 2019. Disponível em: https:// www.who.int/news-room/fact-sheets/detail/mentalhealth-of-older-adults .

5. de Freitas CB, Veloso TCP, da Silva Segundo LP, de Sousa FPG, Galvão BS, Nagaishi CY. Prevalência de depressão entre idosos institucionalizados. Res Soc Dev 2020;9(4):e190943017. Disponível em: https:// doi.org/10.33448/rsd-v9i4.3017.

6. Silva PO, Aguiar BM, Vieira MA, da Costa FM, Carneiro JA. Prevalência de sintomas depressivos e seus fatores associados em idosos atendidos por um centro de referência. Rev Bras Geriatr Gerontol. 2019;22(5):e190088. Disponível em: https://doi. org/10.1590/1981-22562019022.190088 .
7. Skośkiewicz-Malinowska K, Malicka B, Ziętek M, Kaczmarek U. Oral health condition and occurrence of depression in the elderly. Medicine. 2018;97(41):e12490. Disponível em: https://dx.doi. org/10.1097\%2FMD.0000000000012490.

8. Jang JH. Systematic review of the determinants of oral health-related quality of life (OHRQoL) among Korean elderly adults. J Korean Soc Dent Hyg. 2020;20(1):1-9. Disponível em: https://doi. org/10.13065/jksdh.20200001 .

9. Wong FM, Ng YT, Leung WK. Oral Health and Its Associated Factors Among Older Institutionalized Residents: a Systematic Review. Int J Environ Res Public Health. 2019;6(21):1-9. Disponível em: https:// doi.org/10.3390/ijerph16214132.

10. Komiyama T, Ohi T, Miyoshi Y, Murakami T, Tsuboi A, Tomata Y, et al. Relationship between status of dentition and incident functional disability in an elderly Japanese population: prospective cohort study of the Tsurugaya project. J Prosthodont Res. 2018;62(4):443-8. Disponível em: https://doi. org/10.1016/j.jpor.2018.04.003 .

11. Cademartori MG, Gastal MT, Nascimento GG, Demarco FF, Corrêa MB. Is depression associated with oral health outcomes in adults and elders? a systematic review and meta-analysis. Clin Oral Investig. 2018;22(8):2685-2702. Disponível em: https://doi.org/10.1007/s00784-018-2611-y .

12. Silva AER, Kunrath I, Danigno JF, Cascaes AM, Castilhos EDD, Langlois CDO, et al. A Saúde bucal está associada à presença de sintomas depressivos em idosos? Ciênc Saúde Colet. 2019;24(1):181-8. Disponível em: https://doi.org/10.1590/141381232018241.12662017 . 
13. Kunrath I, Silva AER. Oral health and depressive symptoms among older adults: longitudinal study. Aging Ment Health. 2020;12(1):1-7. Disponível em: https://doi.org/10.1080/13607863.2020.1855104 .

14. Guimarães LDA., Brito TA, Pithon KR, Jesus CSD, Souto CS, Souza SJN, et al. Sintomas depressivos e fatores associados em idosos residentes em instituição de longa permanência. Ciênc Saúde Colet. 2019;24(9):3275-82. Disponível em: https://doi. org/10.1590/1413-81232018249.30942017 .

15. Silva e Farias IP, Montenegro L, Wanderley RL, Pontes J, Pereira AC, Almeida L, et al. Physical and psychological states interfere with health-related quality of life of institutionalized elderly: a cross-sectional study. BMC Geriatr. 2020;20(1):1-9. Disponível em: https://doi.org/10.1186/s12877-020-01791-6 .

16. Ferreira NSDA. As pesquisas denominadas" estado da arte". Educ Soc. 2002;23(79):257-72.

17. Mays N, Roberts E, Popay J. Synthesising research evidence. Studying the organisation and delivery of health services: Research methods. [Sem local]: Routledge; 2001.

18. Munn Z, Stern C, Aromataris E, Lockwood C, Jordan, Z. What kind of systematic review should I conduct? A proposed typology and guidance for systematic reviewers in the medical and health sciences. BMC Med Res Methodol. 2018;18(5):1-9. Disponível em: https://bmcmedresmethodol.biomedcentral.com/ articles/10.1186/s12874-017-0468-4 .

19. Kyngäs H. Qualitative research and content analysis. In: Kyngas H, Mikkonen K, Kääriäinen M, editors. The Application of Content Analysis in Nursing Science Research [Internet]. [Sem local]: Springer; 2019 [acesso em 29 mar. 2020]. p. 3-11. Disponível em: https://www.springer.com/gp/ book/9783030301989 .

20. Mota RT, Ferreira Jr. HM, Pereira FS, Vieira MA, Costa SDM. Qualidade de vida em pacientes com câncer de pulmão: scoping review. Rev Bras Geriatr Gerontol. 2019;22(2):e180162. Disponível em: https:// doi.org/10.1590/1981-22562019022.180162

21. Peters M, Godfrey C, McInerney P, Soares CB, Khalil H, Parker D. Methodology for JBI scoping reviews. In: Joanna Briggs Institute Reviewers Manual. [Sem local]: JBI; 2015. p.3-24.

22. de Mello ALSF, Padilha DMP. Oral health care in private and small long-term care facilities: a qualitative study. Gerodontology. 2009;26(1):53-7. Disponível em: https://doi.org/10.1111/j.17412358.2008.00238.x .
23. Ferreira RC, Magalhães CSD, Rocha ES, Schwambach CW, Moreira AN. Saúde bucal de idosos residentes em instituições de longa permanência de Belo Horizonte, Minas Gerais, Brasil. Cad Saúde Pública. 2009;25(11):2375-85. Disponível em: https:// doi.org/10.1590/S0102-311X2009001100008

24. Haumschild MS, Haumschild RJ. The importance of oral health in long-term care. J Am Med Dir Assoc. 2009;10(9):667-71. Disponível em: https://doi. org/10.1016/j.jamda.2009.01.002 .

25. Isaksson R, Becktor JP, Brown A, Laurizohn C, Isaksson S. Oral health and oral implant status in edentulous patients with implant-supported dental prostheses who are receiving long-term nursing care. Gerodontology. 2009;26(4):245-9. Disponível em: https://doi.org/10.1111/j.1741-2358.2009.00275.x .

26. Kim HY, Jang MS, Chung CP, Paik DI, Park YD, Patton LL, et al. Chewing function impacts oral health-related quality of life among institutionalized and community-dwelling Korean elders. Community Dent Oral Epidemiol. 2009;37(5):468-76. Disponível em: https://doi.org/10.1111/j.1600-0528.2009.00489.x .

27. Bush HM, Dickens NE, Henry RG, Durham L, Sallee $\mathrm{N}$, Skelton J, et al. Oral health status of older adults in Kentucky: results from the Kentucky Elder Oral Health Survey. Spec Care Dentist. 2010;30(5):18592. Disponível em: https://doi.org/10.1111/j.17544505.2010.00154.x .

28. de Visschere L, Schols J, Van der Putten GJ, de Baat C, Vanobbergen J. Effect evaluation of a supervised versus non-supervised implementation of an oral health care guideline in nursing homes: a cluster randomised controlled clinical trial. Gerodontology. 2012;29(2):96-106. Disponível em: https://doi. org/10.1111/j.1741-2358.2010.00418.x .

29. Ozkan Y, Özcan M, Kulak Y, Kazazoglu E, Arikan A. General health, dental status and perceived dental treatment needs of an elderly population in Istanbul. Gerodontology. 2011;28(1):28-36. Disponível em: https://doi.org/10.1111/j.1741-2358.2010.00363.x .

30. Mozafari PM, Dalirsani Z, Delavarian Z, Amirchaghmaghi M, Shakeri MT, Esfandyari A, et al. Prevalence of oral mucosal lesions in institutionalized elderly people in Mashhad, Northeast Iran. Gerodontology. 2012;29(2):930-4. Disponível em: https://doi.org/10.1111/j.1741-2358.2011.00588.x .

31. Pretty IA. The life course, care pathways and elements of vulnerability. A picture of health needs in a vulnerable population. Gerodontology. 2014;31(1):18. Disponível em: https://doi.org/10.1111/ger.12092 . 
32. Cocco F, Campus G, Strohmenger L, Ardizzone VC, Cagetti MG. The burden of tooth loss in Italian elderly population living in nursing homes. BMC Geriatr. 2018;18(1):1-9. Disponível em: https://doi. org/10.1186/s12877-018-0760-0 .

33. Machado ACB. Concentração de cortisol salivar, saúde bucal e qualidade de vida de idosos [dissertação]. Araçatuba: Universidade Estadual Paulista; 2018.

34. Recker E, Mendes MSS, Blanchette D, Dawson DV, Cowen H, Hartshorn J, et al. Systemic health characteristics and self-reported xerostomia among nursing facility residents in Iowa-US and Sao PauloBrazil. Braz Dent Sci. 2019;22(1):103-10. Disponível em: https://doi.org/10.14295/bds.2019.v22i1.1677 .

35. Anand S, Ravallion M. Human development in poor countries: on the role of private incomes and public services. J Econ Perspect. 1993;7(1):133-50.

36. Davison TE, Snowdon J, Castle N, McCabe MP, Mellor D, Karantzas G, et al. An evaluation of a national program to implement the Cornell Scale for Depression in Dementia into routine practice in aged care facilities. Int Psychogeriatr. 2012;24(4):1-9. Disponível em: https://doi.org/10.1017/S1041610211002146 .

37. Organização Mundial da Saúde [Internet]. Gênova: WHO; 2019 [acesso em 12 fev. 2021]. Number of nursing and elderly home beds. Disponível em: https://gateway.euro.who.int/en/indicators/hfa_4915101-number-of-nursing-and-elderly-home-beds/ .

38. Drageset J, Eide GE, Ranhoff AH. Anxiety and depression and mortality among cognitively intact nursing home residents with and without a cancer diagnosis: a 5-year follow-up study. Cancer Nurs. 2013;36(4):68-74. Disponível em: https://doi. org/10.1097/NCC.0b013e31826fcb11 .

39. Underwood M, Lamb SE, Eldridge S, Sheehan B, Slowther AM, Spencer A, et al. Exercise for depression in elderly residents of care homes: a cluster-randomised controlled trial. Lancet. 2013;382(9886):41-9. Disponível em: https://doi. org/10.1016/S0140-6736(13)60649-2 .

40. Miller LM, Dieckmann NF, Mattek NC, Lyons KS, Kaye JA. Social activity decreases risk of placement in a long-term care facility for a prospective sample of community-dwelling older adults. Res Gerontol Nurs. 2014;7(3):106-12. Disponível em: https://doi. org/10.3928/19404921-20140110-02 .

41. van Schaik DJ, Dozeman E, van Marwijk HW, Stek ML, Smit F, Beekman AT, et al. Preventing depression in homes for older adults: are effects sustained over 2 years? Int J Geriatr Psychiatry. 2014;29(2):191-7. Disponível em: https://doi. org/10.1002/gps.3989
42. Chesler J, McLaren S, Klein B, Watson S. The effects of playing Nintendo Wii on depression, sense of belonging and social support in Australian aged care residents: a protocol study of a mixed methods intervention trial. BMC Geriatr. 2015;15(1):1-8. Disponível em: https://doi. org/10.1186/s12877-015-0107-z

43. Shokry AAE, Adel MR, Rashad AESA. Educational program to improve quality of life among elderly regarding oral health. Futur Dent J. 2018;4(2):2115. Disponível em: https://doi.org/10.1016/j. fdj.2018.07.002 .

44. Menezes AV, Aguiar ADSD, Alves EF, Quadros LBD, Bezerra PP. Efetividade de uma intervenção fisioterapêutica cognitivo-motora em idosos institucionalizados com comprometimento cognitivo leve e demência leve. Ciênc Saúde Colet. 2016;21(11):3459-67. Disponível em: https://doi. org/10.1590/1413-812320152111.17892015 .

45. Mountford CG, Okonkwo AC, Hart K, Thompson NP. Managing malnutrition in older persons residing in care homes: nutritional and clinical outcomes following a screening and intervention program. J Nutr Gerontol Geriatr. 2016;35(1):52-66. Disponível em: https://doi.org/10.1080/21551197.2015.1131797 .

46. Byers AL, Lui LY, Vittinghoff E, Covinsky KE, Ensrud KE, Taylor B, et al. Burden of depressive symptoms over 2 decades and risk of nursing home placement in older women. J Am Geriatr Soc. 2018;66(10):1895-1901. Disponível em: https://doi. org/10.1111/jgs.15496 .

47. Cassie KM, Cassie WE. Organizational and individual conditions associated with depressive symptoms among nursing home residents over time. Gerontologist. 2012;52(6):812-21. Disponível em: https://doi.org/10.1093/geront/gns059 .

48. Ozaki T, Katsumata Y, Arai A. Association between changes in the use of psychotropic drugs and behavioural and psychological symptoms of dementia over 1 year among long-term care facility residents. Psychogeriatrics. 2019;19(2):126-34. Disponível em: https://doi.org/10.1111/psyg.12374.

49. Bali V, Chatterjee S, Johnson ML, Chen H, Carnahan RM, Aparasu RR. Risk of mortality in elderly nursing home patients with depression using paroxetine. Pharmacotherapy. 2017;37(3):287-96. Disponível em: https://doi.org/10.1002/phar.1898

50. Bomfim FMS, Chiari BM, Roque FP. Factors associated to suggestive signs of oropharyngeal dysphagia in institutionalized elderly women. CoDAS. 2013;25(2):154-63. 